\title{
Rationale for Mass Masking in Controlling the COVID-19 Pandemic
}

\author{
Shing Yau Tam ${ }^{1}$, Victor C. W. Tam ${ }^{1}$, Helen K. W. Law ${ }^{1}$, May Ling Khaw ${ }^{2}$ and \\ Shara W. Y. Lee ${ }^{1 *}$
}

${ }^{1}$ Department of Health Technology and Informatics, Faculty of Health and Social Sciences, The Hong Kong Polytechnic University, Kowloon, China, ${ }^{2}$ Tasmanian School of Medicine, University of Tasmania, Hobart, TAS, Australia

OPEN ACCESS

Edited by:

Bach Tran

Hanoi Medical University, Vietnam

Reviewed by:

Sukanta Sarkar,

Indian Institute of Technology

Ropar, India

Supa Pengpid,

Mahidol University, Thailand

*Correspondence:

Shara W. Y. Lee

shara.lee@polyu.edu.hk

Specialty section:

This article was submitted to

Public Health Policy,

a section of the journal

Frontiers in Public Health

Received: 08 February 2021 Accepted: 03 August 2021

Published: 24 August 2021

Citation:

Tam SY, Tam VCW, Law HKW, Khaw ML and Lee SWY (2021)

Rationale for Mass Masking in

Controlling the COVID-19 Pandemic.

Front. Public Health 9:665708.

doi: 10.3389/fpubh.2021.665708
The rapid spread of the coronavirus disease 2019 (COVID-19) into a global pandemic caught the world unprepared. Previously effective measures for containing disease outbreaks were overwhelmed, necessitating strict controls such as lockdowns or curfews. Among the disease control interventions, community mass masking was one of the highly controversial issues with differing opinions on its indications or effectiveness from different health authorities around the world. Regions where community mass masking was timely introduced were associated with lower transmission rates, and more effective disease control. In this article, we discuss the evidence on the effectiveness, and rationale for community mass masking to prevent the COVID-19 transmission. Areas for further research to define the role of mass masking in light of the COVID-19 pandemic will be suggested. This would help policy makers in formulating mass masking policies.

Keywords: COVID-19, pandemic, public health, public health policy, face mask, infection control, mass masking

\section{INTRODUCTION}

The coronavirus disease 2019 (COVID-19) is the result of infection by the novel severe acute respiratory syndrome coronavirus 2 (SARS-CoV2), making this the third coronavirus to have crossed species and cause severe disease in humans. In the absence of effective vaccine distribution or specific antiviral agents, the only means of limiting transmission is through physical interventions such as mask wearing, physical distancing, and isolation of infectious individuals. Although the routes of transmission via respiratory droplets or aerosols and direct contact are similar to other coronaviruses, COVID-19 has defeated the control measures of many high-income nations with well-funded health infrastructures.

There are several characteristics of the COVID-19 infection that has enabled it to spread efficiently and rapidly developed into a global pandemic. COVID-19 has a wide spectrum of presentations that range from asymptomatic or having only mild symptoms, to systemic illness with multiorgan involvement (1-4). Severity of illness is generally increased in those at a more advanced age, while the younger carriers have only mild symptoms and are consequently able to spread the virus undetected. Another feature of the COVID-19 infection is the long presymptomatic phase, with a median incubation period of 5-6 days and can be as long as 19 days (5-8). Transmission can occur during the latter part of the presymptomatic phase, and $48-62 \%$ of infections were attributed to presymptomatic carriers (9). Moreover, these presymptomatic carriers were able to pass undetected through temperature screening, a previously dependable process for disease control at border checkpoints, and therefore facilitating the rapid spread globally.

As one of the key factors for limiting the transmissibility of COVID-19, community mass masking was one of the public health measures used to mitigate its rapid spread. Initially, this 
practice is not universally adopted, which may account for poorer infection control outcomes for some regions $(10,11)$. The issue of community mass masking at the time of the emergence of COVID-19 has been highly controversial, with conflicting recommendations issued by health authorities around the world. In an earlier guideline during the spread of the pandemic from East to West, the World Health Organization (WHO) conservatively recommended against community mass masking with medical masks due to the lack of concrete evidence, while stressed on reserving the scarce resources for the healthcare workers (12). Although explicable, it seemed to reduce the chance to limit the substantial spreading of the disease in the early stage. This early recommendation was also accompanied by a speculative suggestion of risk compensation with mass masking, leading to the reduced attention in other effective protective behaviors such as performing hand hygiene $(6,12)$. On the contrary, Mantzari et al. (13) systematically reviewed previous studies on wearing mask with hand hygiene in community settings for controlling respiratory infections. They concluded that wearing masks did not reduce the frequency of hand sanitizing while two studies reported higher hand washing rates in the groups with mask wearing.

Notably, the recommendations on mass masking were made based on the evidence from studies on influenza outbreaks, which were not necessarily applicable to the present coronavirus. Given the little amount that was known about the COVID-19 disease and its modes of transmission, many health authorities in Asia (e.g., China, Hong Kong, Singapore etc.) exercised caution by implementing community masking recommendations (12). Subsequent recommendations based on the experience of successful control of early COVID-19 outbreaks in China also advocated for mass masking (14). Generally, Asian societies appear more inclined to wear masks voluntarily when unwell, or for protection during disease outbreaks, given the previous close encounters with epidemics such as the severe acute respiratory syndrome (SARS) in 2003 that predominantly affected Asia. Consequently, compliance with community mass masking recommendations has been high in these regions. In a global survey conducted across 15 countries during the pandemic (April 9-12, 2020) (15), countries with significant proportions of mask wearers included Vietnam (91\%), China (83\%), Italy (81\%), Japan (77\%), and India (76\%). By comparison, this was lower in Western countries including United Kingdom (16\%), Germany (20\%), Australia (21\%), Canada (28\%), and France (34\%).

Evidence from epidemiological data show that with a timely introduction of community mass masking in conjunction with infection prevention and control strategies were more effective in containing the COVID-19 transmission $(10,16,17)$. Escalation of stricter controls such as lockdowns or curfews were subsequently not necessary (10), as exemplified in Taiwan (18). The aim of this article is to discuss the current evidence on the effectiveness, and rationale for community mass masking in preventing COVID-19 transmission. Areas for further research in order to define the role of mass masking in light of the COVID-19 pandemic will be discussed.

\section{RATIONALE FOR MASS MASKING}

Before examining the role of masks in mitigating the COVID19 transmission, its function and basic mechanism of protection should first be considered. A mask provides a semipermeable barrier to limit the passage of particles or substance through it. The relative size of the particle to that of the pores is important. Thus, the purpose of use should be considered before a type of mask is selected. Medically speaking, surgical masks were originally used as source control to prevent contamination by droplets or aerosols containing microbes from the nose and throat of theater staff to the patient's site of operation. The use of surgical masks nowadays also protect staff from potential biohazards encountered in the operating theater, where N95 respirators are also used. Surgical masks and N95 respirators are made of high filtration materials with efficacy of $\geq 95 \%(0.3 \mu \mathrm{m})$. The protective effect of a medical mask is rated based on its filtration efficacy and the ability to provide an airtight fit to the face. When loose fitting and therefore permitting the passage of air around the edges, there is a drop in overall rated filtration efficacy to $<70 \%$, according to the US National (N10SH) N95 test (19). For this reason, the surgical mask is not classified as personal protective equipment (PPE) (20), whereas a well-fitted respirator with a N95 rating fulfills the designation of PPE due to its airtight design, and has a filtration efficacy of $\geq 95 \%$ (19).

When coughing or sneezing, aerosols, and droplet particles are exhaled, which range from 0.1 to $1,000 \mu \mathrm{m}$ in size (11). Aerosols are particles sized $<5 \mu \mathrm{m}$ and they remain airborne for a longer period. The spread of these particles is determined by numerous factors including size, ambient temperature, air movement, and humidity. Droplets may partially evaporate, which cause them to gain more buoyancy and remain airborne for longer to spread over a greater area (11). The smaller submicron $(<1 \mu \mathrm{m})$ aerosol particles are considered to be more dangerous as they can be inhaled and lodged deeper into the lung parenchyma, leading to more severe infections due to interference with gas exchange $(20,21)$.

\section{DISCUSSION}

Recent studies $(21,22)$ have reported that the COVID-19 virus detected in both droplets and aerosols can be propelled further than the recommended one meter of social distancing by the WHO $(13,23)$, or six feet recommendation by the US Centre for Disease Control and Prevention (CDC) (24).

The WHO previously recommended against wearing masks in the community due to a lack of evidence on its effectiveness. However, the evidence underlying this recommendation was drawn from 10 randomized studies on transmission of the influenza, and other human coronaviruses in the community (6).These findings could be not applicable to the transmission of a novel coronavirus which spreads more surreptitiously than influenza due to the presymptomatic carriers and those with mild symptoms that evaded detection. The basic reproductive rate of COVID-19 was estimated to be between 2.2 and 2.7 $(23,25)$, while the rate of the 2009 influenza H1N1 pandemic was estimated to be 1.7 (26). Subsequently in June 2020, the 
WHO guideline was revised to suggest mass masking as part of the comprehensive strategy if there is limited capacity of measures in light of ongoing transmission by asymptomatic carriers in the community settings, and the potential difficulties in maintaining physical distancing and containment measures (27). The importance of mass masking in mitigating COVID-19 pandemic is summarized in Table $\mathbf{1}$.

Reports of transmission by asymptomatic carriers with a high viral load at the initial stage of infection emerged as early as February $2020(5,36)$, with the peak viral load occurring during symptom onset $(37,38)$. A recent meta-analysis of 9 published reports concluded that 15\% of COVID-19 infections were asymptomatic (39), and the incubation period was reported to be 5-6 days, but can be as long as 19 days (5-8). Virus shedding in the upper respiratory tract is high among presymptomatic patients (40), while those with asymptomatic influenza usually have a lower viral load in the upper than the lower respiratory tract (41). This is the new challenge posed by the COVID-19 virus that distinguishes it from most other infectious diseases. Asymptomatic carriers were reported to play a significant role in the community spread. Li et al. (42) estimated that $86 \%$ (95\% CI: 82-90\%) of all infections were undocumented before travel restrictions were implemented in China. Although the transmission rate of undocumented infections was lower than the documented ones, it is estimated to be responsible for $48-62 \%$ of the COVID-19 infections (1). A recent study conducted by Hong et al. (28) reported a significantly higher incidence of COVID-19 among individuals who had close contact with presymptomatic patients without wearing masks ( 19.0 vs. $8.1 \%, p<0.001$ ).

Aerosol particles are smaller, and depending on the physical conditions such as humidity, temperature or wind, can linger in the air for considerably longer compared to droplets (20), thus enabling the virus to spread more efficiently despite physical distancing. The decay rates ranged from 0.4 to $2.27 \%$ per minute and the half-lives $30-177 \mathrm{~min}$ under different conditions with lower relative humidity as a factor for longer half-lives (43). Ultimately, the likelihood of infection with COVID-19 is highly dependent on the contaminating viral dose during transmission $(44,45)$. The rationale for mask wearing is firstly recognized as a source control to minimize the amount of virus exhaled by carriers to the atmosphere. With masking, both the amount and velocity of droplets or aerosols expelled in a breath will be smaller, thus reducing the area of spread. Secondly, the mask will protect healthy individuals from exposure to droplets or aerosols $(29,46)$.

Leung et al. (30) prospectively evaluated the transmission of coronavirus, influenza virus and rhinovirus in droplets and aerosols of the exhaled breath of patients with acute respiratory illnesses. They found that while only some carriers exhaled a detectable viral load, wearing a surgical mask was effective in blocking the transmission of both droplets and aerosols completely. Bae et al. (47) investigated the effectiveness of cotton and surgical masks for preventing virus shedding during coughing by COVID-19 patients. In contrast, they found that neither surgical nor cotton masks could filter the virus effectively, which was attributed to the production of aerosols when coughing. These findings suggest that although the surgical mask is adequate as a source control for asymptomatic carriers in the community, it could be less effective for those who are actively coughing. Symptomatic individuals will need testing and isolation; for source control, the N95 respirator may be required.

Meanwhile, the revised WHO guideline recommended the use of non-medical cloth or homemade masks in the community in order to preserve the stock of medical masks for healthcare workers, and recommended these as a means of source control in community settings but not for prevention (27). The guideline also suggested that wearing cloth masks on public transport, frequent hand hygiene and physical distancing should always be adopted together with cloth masks. Following the revised guideline from the WHO in June 2020 on community mass masking, many countries have adopted this recommendation. However, cloth masks may have varying filtering efficiencies, which are generally lower than that of the medical masks $(48,49)$. There is a lack of solid evidence to support the effectiveness of using cloth mask as a mean of source control to the asymptomatic carriers. A report conducted by Hendrix et al. (31) described 139 clients with cloth face coverings were not infected with COVID19 after contact with two symptomatic hair stylists who also wore face coverings. The hair stylists were eventually tested positive. Yet, one laboratory study reported inferior filtering performance of general masks compared to medical masks (50). Paradoxically, the only clinical outcome study by Zhang et al. (33) reported a higher rate of respiratory illness among hospital staff wearing a cloth mask compared to not wearing a mask for protection at work. The efficacy of using cloth mask in the community setting has never been evaluated in an outcome study for COVID-19.

With the easing of lockdown in many areas, it can be seen that social distancing on its own is inadequate, and the infection rates have increased considerably. Epidemiological data from Asian regions or countries such as China, Hong Kong, Japan, Macau, Singapore, South Korea (14), Taiwan (15) where universal masking was enforced or recommended showed effective control of COVID-19 transmission, and implementation of lockdowns were limited. A number of infections occurred in bars or restaurants where masks were often removed, which emphasizes the importance of this measure (32). A recent study analyzed the trend and mitigation measures in Wuhan, Italy, and New York City from 23 January to 9 May 2020. The findings concluded that the single determinant shaping the pandemic trend was the imposition of mandatory face covering. Surprisingly, other measures such as social distancing failed to suppress transmission (33). Miyazawa et al. (34) reported a strong inverse relationship between the masking rate to the mortality rate from COVID-19; with a predictive power of $69 \%$ based on an analysis of data from 22 countries including 13 western and 9 Asian countries in March 2020. Worby and Chang (35) employed mathematical modeling to evaluate the epidemiological impact of mass masking among general population by considering resource limitations, supply and demand dynamics. They concluded that face masks can reduce total infections and deaths in COVID-19 pandemic even if the protective effect of face masks is limited. In addition, the peak time of the epidemic could be delayed. MacIntyre et al. (16) concluded in a systematic review that wearing a mask in the community was effective for both protection in crowded areas, and as a source control for COVID-19 transmission. They added 
TABLE 1 | Summary of the evidence for mass masking in mitigating COVID-19 pandemic.

\begin{tabular}{|c|c|c|}
\hline Evidence & Theme(s) & References \\
\hline $\begin{array}{l}\text { - Presymptomatic transmission contributed to a significant proportion of the COVID-19 transmission. } \\
\text { - Control strategies, such as community masking, should be adjusted to tackle presymptomatic transmission. }\end{array}$ & $\mathrm{B}$ & (17) \\
\hline - Presymptomatic patients with mask-wearing showed significantly lower transmission to close-contact persons. & A, B & (28) \\
\hline - Surgical face masks reduced viral RNA in respiratory droplets and aerosols from exhaled breath and coughs. & A, C & (30) \\
\hline - Use of cloth face coverings by both symptomatic patients and healthy individual might reduce the risk of transmission. & A & (31) \\
\hline $\begin{array}{l}\text { - The relatively low incidence of COVID-19 in Hong Kong might be contributed by the high compliance of mass masking. } \\
\text { - Transmission clusters in 'mask-off' settings were more prevalent than that in 'mask-on' settings. }\end{array}$ & A & (32) \\
\hline $\begin{array}{l}\text { - Mathematical modeling evaluated that mass masking could reduce total infections and deaths in COVID-19 pandemic. Also, it could delay } \\
\text { the peak time of the epidemic. }\end{array}$ & $\mathrm{D}, \mathrm{E}$ & (35) \\
\hline
\end{tabular}

$A$, reduction in transmissibility; $B$, reduction in presymptomatic spread; $C$, reduction in droplet and aerosol transmission; $D$, reduction in death rate; $E$, delay in peak time of the epidemic.

that mass masking would be more effective if implemented early during an outbreak, which was the case for many Asian countries. Cowling et al. (10) supported this notion by suggesting that nonpharmaceutical interventions including wearing masks among citizens of Hong Kong may contribute to the suppression of local transmission of COVID-19.

A recent study conducted in Hong Kong, a population that is very compliant to community mass masking (94.8\%), reported that the majority of masked individuals (83.7\%) wore surgical masks. From the online questionnaire respondents, $76.3 \%$ reused their masks (51). The low infection rate at the initial stage of outbreak may imply that the reuse of surgical masks in community settings is not as harmful as anticipated, and that the benefits of masking outweigh the risks of reusing masks. A cross-sectional study in Brazil also showed similar percentage of mask reuse (71.1\%) (52). However, it is notable that the efficacy or performance of reused masks is not evaluated. There was one Canadian study evaluating on the reuse of N95 respirators by autoclaving (53). Findings suggested that reusing N95 respirators was feasible in hospitals with successful reuse rate ranging from $48.8 \%$ to $79.6 \%$ in 12 sterilization cycles. The issue of early global medical mask shortage could be addressed by rationing, such as in South Korea (52) and Taiwan (53), or by the provision of free, high efficacy reusable masks in Hong Kong (54) and Singapore (54). Recently, the worldwide supply shortage of medical mask has eased considerably, and this is no longer an issue impeding the implementation of mass masking policy for transmission control in many developed countries.

\section{FURTHER RESEARCH AND DIRECTIONS}

The COVID-19 pandemic has exposed a number of weaknesses in the readiness and competence of many health authorities around the world in handling a novel infectious disease. The
Chinese government resorted to the use of draconian measures to bring control to the transmission, yet many countries remained complacent and underprepared for its malignant spread. Health authorities will benefit from reviewing their capabilities in order to formulate a better epidemic response plan. Although mass masking was recommended based on lessons gained from the experience in China, this was not closely followed in many countries possibly due to the WHO's earlier guidelines against community masking. Reports of presymptomatic carriers and also transmission via aerosol route were largely disregarded. Prompt and continuous re-evaluation and adoption of newly evident recommendations by health authorities would be essential to savagely control the outbreak of the novel virus.

Future research should be directed to address these deficits. The effectiveness of repeated used cloth masks in limiting the spread of respiratory diseases, which address the shortage of disposable surgical masks. The filtration efficiency and safety test using a standardized protocol worldwide should be adopted to facilitate interpretation and comparison. The reusable copper mask that incorporates antimicrobial properties to its filter is an example of innovation, spurred by the mask shortage in Hong Kong (55). The mask complies with the American Society of Testing and Materials (ASTM) F2100 Level 1 standard and can be used in community settings. While Gilbert et al. (56) proposed an inexpensive ultraviolet system for filtering facepiece respirator decontamination that may ease the respirator demand. Efforts should be directed to the design of masks made to improve protection against aerosols that can be easily mass-produced, and safely reused by appropriate decontamination devices.

\section{CONCLUSIONS}

We have summarized and discussed the evidence on community mass masking as a public health measure for controlling the 
COVID-19 transmission. Regions where mass masking was timely introduced were effective with disease control (10), potentially leading to fewer cases of mortality (57). As a source control, the surgical mask is effective, but only if carriers are asymptomatic and do not cough. With easing of lockdowns, the role of mass masking becomes more vital and remains an essential measure for controlling transmission. Lastly, the most important lesson to learn from the COVID-19 pandemic is to focus on better preparation, communication, and international cooperation to effectively limit the spread of emerging infectious diseases in the future.

\section{DATA AVAILABILITY STATEMENT}

The original contributions presented in the study are included in the article/supplementary material, further inquiries can be directed to the corresponding author/s.

\section{REFERENCES}

1. Wiersinga WJ, Rhodes A, Cheng AC, Peacock SJ, Prescott HC. Pathophysiology, transmission, diagnosis, and treatment of coronavirus disease 2019 (COVID-19): a review. JAMA. (2020) 324:782-93. doi: 10.1001/jama.2020.12839

2. Long B, Brady WJ, Koyfman A, Gottlieb M. Cardiovascular complications in COVID-19. Am J Emerg Med. (2020) 38:15047. doi: 10.1016/j.ajem.2020.04.048

3. Mao R, Qiu Y, He JS, Tan JY, Li XH, Liang J, et al. Manifestations and prognosis of gastrointestinal and liver involvement in patients with COVID19: a systematic review and meta-analysis. Lancet Gastroenterol Hepatol. (2020) 5:667-78. doi: 10.1016/S2468-1253(20)30126-6

4. Rodriguez-Morales AJ, Cardona-Ospina JA, Gutiérrez-Ocampo E, VillamizarPeña R, Holguin-Rivera Y, Escalera-Antezana JP, et al. Clinical, laboratory and imaging features of COVID-19: a systematic review and meta-analysis. Travel Med Infect Dis. (2020) 34:101623. doi: 10.1016/j.tmaid.2020.101623

5. Bai Y, Yao L, Wei T, Tian F, Jin DY, Chen L, et al. Presumed asymptomatic carrier transmission of COVID-19. JAMA. (2020) 323:14067. doi: 10.1001/jama.2020.2565

6. World Health Organization. Advice on the Use of Masks in the Context of COVID-19: Interim Guidance 6 April 2020. (2020). Available online at: https:// apps.who.int/iris/handle/10665/331693 (accessed February 7, 2021).

7. Pan X, Chen D, Xia Y, Wu X, Li T, Ou X, et al. Asymptomatic cases in a family cluster with SARS-CoV-2 infection. Lancet Infect Dis. (2020) 20:4101. doi: 10.1016/S1473-3099(20)30114-6

8. Yu P, Zhu J, Zhang Z, Han Y. A familial cluster of infection associated with the 2019 novel coronavirus indicating possible person-to-person transmission during the incubation period. J Infect Dis. (2020) 221:175761. doi: 10.1093/infdis/jiaa077

9. Ganyani T, Kremer C, Chen D, Torneri A, Faes C, Wallinga J, et al. Estimating the generation interval for COVID-19 based on symptom onset data. medRxiv [Preprint]. (2020) 25:2000257. doi: 10.2807/1560-7917.ES.2020.25.29.20 01269

10. Cowling BJ, Ali ST, Ng TWY, Tsang TK, Li JCM, Fong MW, et al. Impact assessment of non-pharmaceutical interventions against coronavirus disease 2019 and influenza in Hong Kong: an observational study. Lancet Public Health. (2020) 5:e279-88. doi: 10.1016/S2468-2667(20)30090-6

11. Prather KA, Wang CC, Schooley RT. Reducing transmission of SARS-CoV-2. Science. (2020) 368:1422-4. doi: 10.1126/science.abc6197

12. World Health Organization. Advice on the Use of Masks the Community, During Home Care and in Health Care Settings in the Context of COVID19: Interim Guidance 19 March 2020. (2020). Available online at: https://apps. who.int/iris/handle/10665/331493 (accessed February 7, 2021).

\section{AUTHOR CONTRIBUTIONS}

ST and VT performed the literature search. ST, VT, MK, and SL collected information and drafted the manuscript. SL supervised the study and edited the manuscript. ST, VT, HL, and MK edited the manuscript. All authors contributed to the article and approved the submitted version.

\section{FUNDING}

This study was supported by departmental and institutional funds from The Hong Kong Polytechnic University.

\section{ACKNOWLEDGMENTS}

We thank Dr. K. S. Khaw for his professional advice with manuscript preparation.

13. Mantzari E, Rubin GJ, Marteau TM. Is risk compensation threatening public health in the covid-19 pandemic? BMJ. (2020) 370:m2913. doi: 10.1136/bmj.m2913

14. Zhang J, Litvinova M, Wang W, Wang Y, Deng X, Chen X, et al. Evolving epidemiology and transmission dynamics of coronavirus disease 2019 outside Hubei province, China: a descriptive and modelling study. Lancet Infect Dis. (2020) 20:793-802. doi: 10.1016/S1473-3099(20)30230-9

15. Bricker D. More People Say They're Wearing Masks to Protect Themselves from COVID-19 Since March Paris: IPSOS. (2020). Available online at: https:// www.ipsos.com/sites/default/files/ct/news/documents/2020-04/coronaviruswave-8-april-16-pr-ipsos.pdf (accessed April 16, 2020).

16. MacIntyre CR, Chughtai AA. A rapid systematic review of the efficacy of face masks and respirators against coronaviruses and other respiratory transmissible viruses for the community, healthcare workers and sick patients. Int J Nurs Stud. (2020) 108:103629. doi: 10.1016/j.ijnurstu.2020.103629

17. He X, Lau EHY, Wu P, Deng X, Wang J, Hao X, et al. Temporal dynamics in viral shedding and transmissibility of COVID-19. Nat Med. (2020) 26:6725. doi: 10.1038/s41591-020-0869-5

18. Kirby J. What We Can Learn from the "Second Wave" of Coronavirus Cases in Asia: Vox. (2020). Available online at: https://www.vox.com/2020/4/17/ 21213787/coronavirus-asia-waves-hong-kong-singapore-taiwan (accessed April 17, 2020).

19. 3M Personal Safety Division. Respirators and Surgical Masks: A Comparison. (2020). Available online at: http://multimedia.3m.com/mws/media/957730O/ respirators-and-surgical-masks-contrast-technical-bulletin.pdf (accessed May 30, 2020).

20. Tellier R, Li Y, Cowling BJ, Tang JW. Recognition of aerosol transmission of infectious agents: a commentary. BMC Infect Dis. (2019) 19:101. doi: 10.1186/s12879-019-3707-y

21. Buonanno G, Stabile L, Morawska L. Estimation of airborne viral emission: quanta emission rate of SARS-CoV-2 for infection risk assessment. Environ Int. (2020) 141:105794. doi: 10.1016/j.envint.2020.105794

22. Mittal R, Ni R, Seo J-H. The flow physics of COVID-19. J Fluid Mech. (2020) 894:F2. doi: 10.1017/jfm.2020.330

23. Wu JT, Leung K, Leung GM. Nowcasting and forecasting the potential domestic and international spread of the 2019-nCoV outbreak originating in Wuhan, China: a modelling study. Lancet. (2020) 395:689-97. doi: 10.1016/S0140-6736(20)30260-9

24. Centers for Disease Control and Prevention. Protect Yourself. US (2020). Available online at: https://www.cdc.gov/coronavirus/2019-ncov/preventgetting-sick/prevention.html (accessed April 24, 2020).

25. Li Q, Guan X, Wu P, Wang X, Zhou L, Tong Y, et al. Early transmission dynamics in Wuhan, China, of novel coronavirus-infected pneumonia. N Engl J Med. (2020) 382:1199-207. doi: 10.1056/NEJMoa2001316 
26. Balcan D, Hu H, Goncalves B, Bajardi P, Poletto C, Ramasco JJ, et al. Seasonal transmission potential and activity peaks of the new influenza A (H1N1): a Monte Carlo likelihood analysis based on human mobility. BMC Med. (2009) 7:45. doi: 10.1186/1741-7015-7-45

27. World Health Organization. Advice on the Use of Masks in the Context of COVID-19: Interim Guidance 5 June 2020. (2020). Available online at: https:// apps.who.int/iris/bitstream/handle/10665/332293/WHO-2019-nCov-IPC_ Masks-2020.4-eng.pdf? sequence $=1$ \&isAllowed $=y \quad$ (accessed February 7, 2021).

28. Hong LX, Lin A, He ZB, Zhao HH, Zhang JG, Zhang C, et al. Mask wearing in pre-symptomatic patients prevents SARS-CoV-2 transmission: an epidemiological analysis. Travel Med Infect Dis. (2020) 36:101803. doi: 10.1016/j.tmaid.2020.101803

29. Chan JF-W, Yuan S, Zhang AJ, Poon VK-M, Chan CC-S, Lee AC-Y, et al. Surgical mask partition reduces the risk of non-contact transmission in a golden Syrian hamster model for coronavirus disease 2019 (COVID-19). Clin Infect Dis. (2020). doi: 10.1093/cid/ciaa644

30. Leung NHL, Chu DKW, Shiu EYC, Chan KH, McDevitt JJ, Hau BJP, et al. Respiratory virus shedding in exhaled breath and efficacy of face masks. Nat Med. (2020) 26:676-80. doi: 10.1038/s41591-020-0843-2

31. Hendrix MJ, Walde C, Findley K, Trotman R. Absence of apparent transmission of SARS-CoV-2 from two stylists after exposure at a hair salon with a universal face covering policy - Springfield, Missouri, May 2020. MMWR Morb Mortal Wkly Rep. (2020) 69:930-2. doi: 10.15585/mmwr.mm6928e2

32. Cheng VC, Wong SC, Chuang VW, So SY, Chen JH, Sridhar S, et al. The role of community-wide wearing of face mask for control of coronavirus disease 2019 (COVID-19) epidemic due to SARS-CoV-2. J Infect. (2020) 81:107-14. doi: 10.1016/j.jinf.2020.04.024

33. Zhang $\mathrm{R}$, Li Y, Zhang AL, Wang Y, Molina MJ. Identifying airborne transmission as the dominant route for the spread of COVID-19. Proc Natl Acad Sci USA. (2020) 117:14857-63. doi: 10.1073/pnas.2009637117

34. Miyazawa D, Kaneko G. Face mask wearing rate predicts country's COVID-19 death rates. medRxiv [Preprint]. (2020). doi: 10.1101/2020.06.22.20137745

35. Worby CJ, Chang HH. Face mask use in the general population and optimal resource allocation during the COVID-19 pandemic. Nat Commun. (2020) 11:4049. doi: 10.1038/s41467-020-17922-x

36. Zou L, Ruan F, Huang M, Liang L, Huang H, Hong Z, et al. SARS-CoV-2 viral load in upper respiratory specimens of infected patients. N Engl J Med. (2020) 382:1177-9. doi: 10.1056/NEJMc2001737

37. To KK-W, Tsang OT-Y, Yip CC-Y, Chan K-H, Wu T-C, Chan JM-C, et al. Consistent detection of 2019 novel coronavirus in saliva. Clin Infect Dis. (2020) 71:841-3. doi: 10.1093/cid/ciaa149

38. Wang FS, Zhang C. What to do next to control the 2019-nCoV epidemic? Lancet. (2020) 395:391-3. doi: 10.1016/S0140-6736(20)30300-7

39. Byambasuren O, Cardona M, Bell K, Clark J, McLaws M-L, Glasziou P. Estimating the extent of asymptomatic COVID-19 and its potential for community transmission: systematic review and meta-analysis. medRxiv [Preprint]. (2020). doi: 10.2139/ssrn.3586675

40. Wölfel R, Corman VM, Guggemos W, Seilmaier M, Zange S, Müller MA, et al. Virological assessment of hospitalized patients with COVID-2019. Nature. (2020) 581:465-9. doi: 10.1038/s41586-020-2196-x

41. Ip DK, Lau LL, Leung NH, Fang VJ, Chan KH, Chu DK, et al. Viral shedding and transmission potential of asymptomatic and paucisymptomatic influenza virus infections in the community. Clin Infect Dis. (2017) 64:73642. doi: 10.1093/cid/ciw841

42. Li R, Pei S, Chen B, Song Y, Zhang T, Yang W, et al. Substantial undocumented infection facilitates the rapid dissemination of novel coronavirus (SARS-CoV2). Science. (2020) 368:489. doi: 10.1126/science.abb3221

43. Smither SJ, Eastaugh LS, Findlay JS, Lever MS. Experimental aerosol survival of SARS-CoV-2 in artificial saliva and tissue culture media at medium and high humidity. Emerg Microbes Infect. (2020) 9:14157. doi: 10.1080/22221751.2020.1777906

44. Dalton CB, Corbett SJ, Katelaris AL. Pre-emptive low cost social distancing and enhanced hygiene implemented before local COVID-19 transmission could decrease the number and severity of cases. Med J Austral. (2020) 212:1. doi: 10.2139/ssrn.3549276
45. Douglas MG, Kocher JF, Scobey T, Baric RS, Cockrell AS. Adaptive evolution influences the infectious dose of MERS-CoV necessary to achieve severe respiratory disease. Virology. (2018) 517:98-7. doi: 10.1016/j.virol.2017.12.006

46. Konda A, Prakash A, Moss GA, Schmoldt M, Grant GD, Guha S. Aerosol filtration efficiency of common fabrics used in respiratory cloth masks. ACS Nano. (2020) 14:6339-47. doi: 10.1021/acsnano.0c03252

47. Bae S, Kim M-C, Kim JY, Cha H-H, Lim JS, Jung J, et al. Effectiveness of surgical and cotton masks in blocking SARS-CoV-2: a controlled comparison in 4 patients. Ann Int Med. (2020) 173:W22-3. doi: 10.7326/M20-1342

48. Rengasamy S, Eimer B, Shaffer RE. Simple respiratory protection-evaluation of the filtration performance of cloth masks and common fabric materials against 20-1000 nm size particles. Ann Occupat Hyg. (2010) 54:78998. doi: 10.1093/annhyg/meq044

49. Shakya KM, Noyes A, Kallin R, Peltier RE. Evaluating the efficacy of cloth facemasks in reducing particulate matter exposure. J Expo Sci Environ Epidemiol. (2017) 27:352-7. doi: 10.1038/jes.2016.42

50. Jung H, Kim JK, Lee S, Lee J, Kim J, Tsai P, et al. Comparison of filtration efficiency and pressure drop in anti-yellow sand masks, quarantine masks, medical masks, general masks, and handkerchiefs. Aerosol Air Qual Res. (2014) 14:991-1002. doi: 10.4209/aaqr.2013.06.0201

51. Tam VCW, Tam SY, Poon WK, Law HKW, Lee SWY. A reality check on the use of face masks during the COVID-19 outbreak in Hong Kong. EClinicalMedicine. (2020) 22:100356. doi: 10.1016/j.eclinm.2020.1 00356

52. Pereira-Ávila FMV, Lam SC, Góes FGB, Gir E, Pereira-Caldeira NMV, Teles SA, et al. Factors associated with the use and reuse of face masks among Brazilian individuals during the COVID-19 pandemic. Rev Lat Am Enfermagem. (2020) 28:e3360. doi: 10.1590/1518-8345.4604.3360

53. Czubryt MP, Stecy T, Popke E, Aitken R, Jabusch K, Pound R, et al. N95 mask reuse in a major urban hospital: COVID-19 response process and procedure. J Hosp Infect. (2020) 106:277-82. doi: 10.1016/j.jhin.2020. 07.035

54. Government of Singapore. How to Redeem the New Reusable Mask. Singapore (2020). Available online at: https://www.gov.sg/article/when-should-i-weara-mask (accessed May 23, 2020).

55. The Government of the Hong Kong Special Administrative Region. What Is CuMask+. (2020). Available online at: https://www.qmask.gov.hk/about/ (accessed June 2, 2020).

56. Gilbert RM, Donzanti MJ, Minahan DJ, Shirazi J, Hatem CL, Hayward-Piatkovskyi B, et al. Mask reuse in the COVID19 pandemic: creating an inexpensive and scalable ultraviolet system for filtering facepiece respirator decontamination. Glob Health Sci Pract. (2020) 8:582-95. doi: 10.9745/GHSP-D-2000218

57. Kai D, Goldstein G-P, Morgunov A, Nangalia V, Rotkirch A. Universal Masking Is Urgent in the COVID-19 Pandemic: SEIR and Agent Based Models, Empirical Validation, Policy Recommendations. (2020). Available online at: https://ui. adsabs.harvard.edu/abs/2020arXiv200413553K (accessed February 7, 2021).

Conflict of Interest: The authors declare that the research was conducted in the absence of any commercial or financial relationships that could be construed as a potential conflict of interest.

Publisher's Note: All claims expressed in this article are solely those of the authors and do not necessarily represent those of their affiliated organizations, or those of the publisher, the editors and the reviewers. Any product that may be evaluated in this article, or claim that may be made by its manufacturer, is not guaranteed or endorsed by the publisher.

Copyright (c) 2021 Tam, Tam, Law, Khaw and Lee. This is an open-access article distributed under the terms of the Creative Commons Attribution License (CC BY). The use, distribution or reproduction in other forums is permitted, provided the original author(s) and the copyright owner(s) are credited and that the original publication in this journal is cited, in accordance with accepted academic practice. No use, distribution or reproduction is permitted which does not comply with these terms. 